\title{
Differential diagnostic value of CD5 and CD117 expression in thoracic tumors: $A$ large scale study of 1465 non-small cell lung cancer cases
}

\author{
Mark Kriegsmann ${ }^{1 *}$, Thomas Muley ${ }^{2,5}$, Alexander Harms ${ }^{1}$, Luca Tavernar ${ }^{1}$, Torsten Goldmann ${ }^{3,6}$,
} Hendrik Dienemann ${ }^{4,5}$, Esther Herpel ${ }^{1+}$ and Arne Warth ${ }^{1,5+}$

\begin{abstract}
Background: Thoracic pathologists are frequently faced with tissue specimens from intrathoracic/mediastinal tumors. Specifically the differentiation between thymic and pulmonary squamous cell carcinomas (SqCC) can be challenging. In order to clarify the differential diagnostic value of CD5 and CD117 in this setting, we performed a large scale expression study of both markers in 1465 non-small cell lung cancer (NSCLC) cases.

Methods: Tissue microarrays of formalin-fixed paraffin-embedded resection specimens of 1465 NSCLC were stained with antibodies against CD117 and CD5. Positivity of both markers was correlated with clinicopathological variables.

Results: CD117 was positive in 145 out of 1457 evaluable cases (9.9\%) and CD5 was positive in 133 out of 1427 evaluable cases (9.3\%). 28 cases (1.9\%) showed coexpression of CD117 and CD5. Among the 145 cases that were positive for CD117, 97 (66.8 \%) were adenocarcinomas (ADC), 34 (23.4 \%) were SqCC, 5 (3.4 \%) were adenosquamous carcinomas (ADSqCC), 8 (5.5 \%) were large cell carcinomas (LC), and one (0.6 \%) was a pleomorphic carcinoma (PC). In the CD5 positive group consisting of 133 cases, 123 (92.4\%) were ADC, 0 (0 \%) were SqCC, 4 (3.0 \%) were ADSqCC, 3 (2.2\%) LC and 3 (2.2\%) were PC. None of the 586 SqCC showed expression of CD5. No association of CD117- or CD5 positivity to patients' age, pathological stages or to T-, N-, or Mcategories was observed.

Conclusions: A substantial subset of NSCLC exhibit positivity of CD117 and CD5. Since CD5 expression was not observed in pulmonary SqCC, but is expressed in the majority of thymic squamous cell carcinomas, the application of this immunomarker is a valuable tool in the differential diagnosis of thoracic neoplasms.
\end{abstract}

Keywords: NSCLC, Thymic carcinoma, CD5, CD117, Mediastinal mass

\section{Background}

Comprehensive morphological and immunohistochemical subtyping of tumors is of growing importance for therapy selection and propelled the concept of a tumor-specific, individualized treatment. For nonsmall cell lung cancer (NSCLC) the current WHO Classification [1] therefore extended the concept of

\footnotetext{
* Correspondence: mark.kriegsmann@med.uni-heidelberg.de

${ }^{\dagger}$ Equal contributors

'Institute of Pathology, Heidelberg University, Im Neuenheimer Feld 224, Heidelberg, Germany

Full list of author information is available at the end of the article
}

immunophenotyping from biopsies to resection specimens. However, the vast majority of NSCLC cases remain non-resectable at initial diagnosis where often only small biopsies or even cytology material is available. Thoracic pathologists are frequently faced with tissue specimens from central tumors with mediastinal involvement. In this setting it is particularly challenging to distinguish pulmonary from thymic primaries [2]. With approximately $80 \%[3,4]$ the most common phenotype of malignant thymic tumors is squamous cell carcinoma; thus, the differentiation of squamous cell carcinoma of the lung $(\mathrm{SqCC})$ is

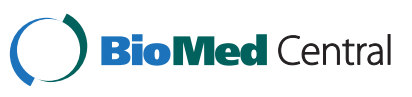

(c) 2015 Kriegsmann et al. Open Access This article is distributed under the terms of the Creative Commons Attribution 4.0 International License (http://creativecommons.org/licenses/by/4.0/), which permits unrestricted use, distribution, and reproduction in any medium, provided you give appropriate credit to the original author(s) and the source, provide a link to the Creative Commons license, and indicate if changes were made. The Creative Commons Public Domain Dedication waiver (http://creativecommons.org/publicdomain/zero/1.0/) applies to the data made available in this article, unless otherwise stated. 
challenging. However, differentiation of both is of high clinical importance since therapies differ substantially [5] and thymic carcinomas are associated with a better prognosis [6]. Since thymic tumors show a high variability with respect to their phenotype [7], but occur with low frequency, the establishment of specific immunomarkers for the differential diagnostic setting requires large scale phenotyping approaches. CD117 $[8,9]$ and CD5 [10-13] are well-known diagnostic markers for thymic carcinomas and are frequently used to separate thymomas from thymic squamous cell carcinomas. However, data on both markers in NSCLC is limited. In order to clarify the differential diagnostic value of CD117 and CD5 to separate between pulmonary and thymic primaries, we performed a large scale expression study of both markers in 1465 NSCLC and correlated the findings with common clinicopathological variables.

\section{Methods}

\section{Cohort characteristics and TMA construction}

Formalin-fixed paraffin-embedded specimens of NSCLC resected from 2002 to 2010 at the Thoraxklinik at Heidelberg University were extracted from the archive of the Institute of Pathology, Heidelberg University with the support of the tissue bank of the National Center for Tumor Diseases (NCT: project: \# 1283). Tissues were used in accordance with the ethical regulations of the NCT tissue bank established by the local ethics committee. A cohort of 1465 patients was identified for TMA construction. Diagnoses were made according to the recommendations of the World Health Organization classification for lung cancer 2015 [1, 14]. Prior to TMA construction, a hematoxylin and eosin (H\&E)-stained slide of each block was analyzed in order to select representative tumor-containing regions. A TMA machine (AlphaMetrix Biotech, Rödermark, Germany) was used to extract a tandem $1.0 \mathrm{~mm}$ cylindrical core sample from each tissue donor block. The cohort characteristics are summarized in Table 1.

\section{Immunohistochemistry}

Immunohistochemistry (IHC) was performed with commercially available antibodies (Additional file 1: Table S1) and was applied according to quality-controlled protocols that are regularly evaluated in round robin trials (http://www.nordiqc.org). In brief, TMA slides were deparaffinized and pretreated with antigen retrieval buffer. Subsequent steps were performed on an immunostaining device (Ventana BenchMark Ultra, Tuscon, USA). Pretreatment and dilution specifications are summarized in Additional file 1: Table S1. Evaluation of IHC was performed blinded to the resection specimen diagnoses, according to a dichotomous scoring scheme [15].
$1 \%$ stained tumor cells were considered to indicate positivity. Each tumor from a patient was represented by two cores on the respective TMA; both cores were analyzed together.

\section{Statistics}

Data analysis was performed using $\mathrm{R}$ statistical software (v. 3.2.2) and RStudio (v. 0.98.507). Barplots were created with the ggplot2-package (v. 1.0.1) and dependencies. $T$-test was applied to examine differences in age. Fisher's exact test of independence or Freeman-Halton test was applied to compare between histotypes, T-, Nand $\mathrm{M}$ - categories and clinical stage. A $p$-value $<0.01$ was considered statistically significant.

\section{Results}

\section{Patient characteristics}

Clinicopathological data of 1465 NSCLC cases (stage IAIV) were analyzed. The median age at diagnosis was 64 years (range: $30-89$ years). The majority of patients were male $(69.3 \%) .711$ patients were diagnosed with adenocarcinoma (ADC; $48.5 \%$ ), 586 patients with squamous cell carcinoma (SqCC; $40.0 \%$ ), 55 patients with adenosquamous carcinoma (AdSqCC; $3.8 \%$ ), 71 patients with large cell carcinoma (LC; $4.8 \%$ ) and 42 patients with pleomorphic carcinoma (PC; $2.9 \%$ ), respectively. Neither CD117 positivity, nor CD5 positivity correlated to patients' age $(p=0.79$ and $p=0.13)$, any T- $(p=0.07$ and $p=0.02), \mathrm{N}-(p=0.16$ and $p=0.03)$ and M-categories $(p=0.42$ and $p=0.77)$ or clinical stage $(p=0.32$ and $p=0.05)$.

\section{Expression of CD117 and CD5 in NSCLC}

In 46 patients (3.1\%) CD117 and/or CD5 could not be evaluated because there were either no vital tumor cells or lost tissue cores during the staining process.

Both, CD117 and CD5 showed diffuse membranous and cytoplasmic positivity (Fig. 1). CD117 was positive in 145 out of 1457 evaluable cases (9.9\%) and CD5 was positive in 133 out of 1427 evaluable cases (9.3\%). 28 cases $(1.9 \%)$ showed coexpression of CD117 and CD5 (Table 1).

Among the 145 CD117 positive cases, 97 (66.8\%) were $\mathrm{ADC}, 34$ (23.4\%) were SqCC, 5 (3.4 \%) were ADSqCC, 8 (5.5 \%) were LC, and one (0.6\%) was a PC.

The frequency of CD117 positive tumors was higher in ADC than in any other histotype $(p<0.01)$.

In the CD5 positive group consisting of 133 cases, 123 $(92.5 \%)$ were $\mathrm{ADC}, 0$ (0 \%) were $\mathrm{SqCC}, 4$ (3.0 \%) were ADSqCC, 3 (2.2\%) were LC and 3 (2.2\%) were PC. The frequency of $\mathrm{CD} 5$ positive tumors was higher in ADC than in any other histotype $(p<0.01)$. No SqCC showed expression of CD5. 
Table 1 Basic clinicopathological characteristics of the analyzed NSCLC cohort

\begin{tabular}{|c|c|c|c|c|c|c|c|c|c|c|}
\hline \multirow[t]{2}{*}{ Clinicopathological Variables } & & \multicolumn{2}{|l|}{ CD117 } & \multirow[t]{2}{*}{$p$-value } & \multicolumn{2}{|l|}{ CD5 } & \multirow[t]{2}{*}{$p$-value } & \multicolumn{2}{|c|}{ CD117/CD5 } & \multirow[t]{2}{*}{$p$-value } \\
\hline & & positive & negative & & positive & negative & & positive & negative & \\
\hline Mean age at surgery & 63 years & 63 years & 63 years & $p=0.79$ & 62 years & 63 years & $p=0.13$ & 61 years & 63 years & $p=0.25$ \\
\hline \multicolumn{11}{|l|}{ Patient gender } \\
\hline Male & $1016(69.3 \%)$ & 89 & 921 & $p=0.03$ & 76 & 910 & $p<0.01$ & 14 & 852 & $p<0.01$ \\
\hline Female & $448(30.5 \%)$ & 56 & 390 & & 57 & 383 & & 10 & 356 & \\
\hline \multicolumn{11}{|l|}{ Histology } \\
\hline Adenocarcinoma & $711(48.5 \%)$ & 97 & 611 & $p<0.01$ & 123 & 564 & $p<0.01$ & 26 & 685 & $p<0.01$ \\
\hline Squamous Cell Carcinoma & $586(40.0 \%)$ & 34 & 548 & & 0 & 575 & & 0 & 586 & \\
\hline Adenosquamous Carcinoma & $55(3.8 \%)$ & 5 & 49 & & 4 & 50 & & 1 & 54 & \\
\hline Large Cell Carcinoma & $71(4.8 \%)$ & 8 & 63 & & 3 & 67 & & 1 & 70 & \\
\hline Pleomorphic Carcinoma & $42(2.9 \%)$ & 1 & 41 & & 3 & 38 & & 0 & 42 & \\
\hline \multicolumn{11}{|l|}{ TNM-Classification } \\
\hline pT1a & 125 (8.5 \%) & 21 & 102 & $p=0.07$ & 16 & 103 & $p=0.02$ & 4 & 121 & $p=0.10$ \\
\hline pT1b & $158(10.8 \%)$ & 19 & 138 & & 20 & 135 & & 5 & 153 & \\
\hline pT2a & 641 (43.8 \%) & 62 & 578 & & 64 & 554 & & 15 & 626 & \\
\hline pT2b & $245(16.7 \%)$ & 22 & 221 & & 16 & 228 & & 2 & 243 & \\
\hline pT3 & 248 (16.9 \%) & 17 & 230 & & 13 & 230 & & 1 & 247 & \\
\hline pT4 & 48 (3.3 \%) & 4 & 43 & & 4 & 44 & & 1 & 47 & \\
\hline pNO & 755 (51.5 \%) & 83 & 667 & $p=0.16$ & 80 & 651 & $p=0.03$ & 16 & 739 & $p=0.57$ \\
\hline $\mathrm{pN} 1$ & $353(24.0 \%)$ & 27 & 324 & & 15 & 330 & & 2 & 351 & \\
\hline $\mathrm{pN} 2$ & 351 (23.9\%) & 35 & 315 & & 38 & 309 & & 10 & 341 & \\
\hline $\mathrm{pN3}$ & $6(0.4 \%)$ & 0 & 6 & & 0 & 4 & & 0 & 6 & \\
\hline $\mathrm{pMO}$ & 1426 (97.3 \%) & 143 & 1275 & $p=0.42$ & 129 & 1261 & $p=0.77$ & 27 & 1399 & $p=0.53$ \\
\hline pM1a & $6(0.4 \%)$ & 0 & 6 & & 2 & 4 & & 1 & 5 & \\
\hline $\mathrm{pM} 1 \mathrm{~b}$ & $33(2.3 \%)$ & 2 & 31 & & 2 & 29 & & 0 & 33 & \\
\hline \multicolumn{11}{|l|}{ Clinical stage } \\
\hline I A & 190 (12.9\%) & 25 & 162 & $p=0.32$ & 25 & 156 & $p=0.05$ & 6 & 184 & $p=0.48$ \\
\hline IB & $344(23.5 \%)$ & 32 & 312 & & 38 & 293 & & 7 & 337 & \\
\hline$\| \mathrm{A}$ & 277 (18.9\%) & 28 & 248 & & 15 & 259 & & 3 & 274 & \\
\hline$\| B$ & $165(11.3 \%)$ & 18 & 145 & & 11 & 152 & & 1 & 164 & \\
\hline$\| I I A$ & 422 (28.8 \%) & 40 & 380 & & 39 & 376 & & 11 & 411 & \\
\hline$\| \mathrm{IIB}$ & $29(1.9 \%)$ & 0 & 29 & & 2 & 25 & & 0 & 29 & \\
\hline IV & 38 (2.6 \%) & 2 & 36 & & 3 & 33 & & 0 & 38 & \\
\hline
\end{tabular}

In the 28 cases that exhibited coexpression of CD117 and $\mathrm{CD} 5,26$ were $\mathrm{ADC}(92.8 \%)$, one was $\mathrm{ADSqCC}$ (3.5\%) and one was a LC (3.5\%). Again, CD5/CD117 coexpression was significantly more frequent in $\mathrm{ADC}$ than in any other histotype $(p<0.01)$. CD5 and CD5/ CD117 positive patients were more often male $(p<0.01)$. Application of the immunoreactive score by Remmele et Stegner [16] could not improve the diagnostic power of any category. A summary of IHC findings is provided in Table 1 and Fig. 2.

\section{Discussion}

This is the largest study analyzing the expression of CD5 and CD117 in NSCLC. We demonstrate that positivity for both markers is evident in approximately $10 \%$ of all NSCLC and is predominantly found in ADC. While coexpression of CD5 and CD117 is rare and also predominates in ADC, the coexpression of both markers was not detected in SqCC. Likewise none of the SqCC showed CD5 expression. Thus, a marker panel including CD5 and CD117 is helpful in the differential diagnosis of 


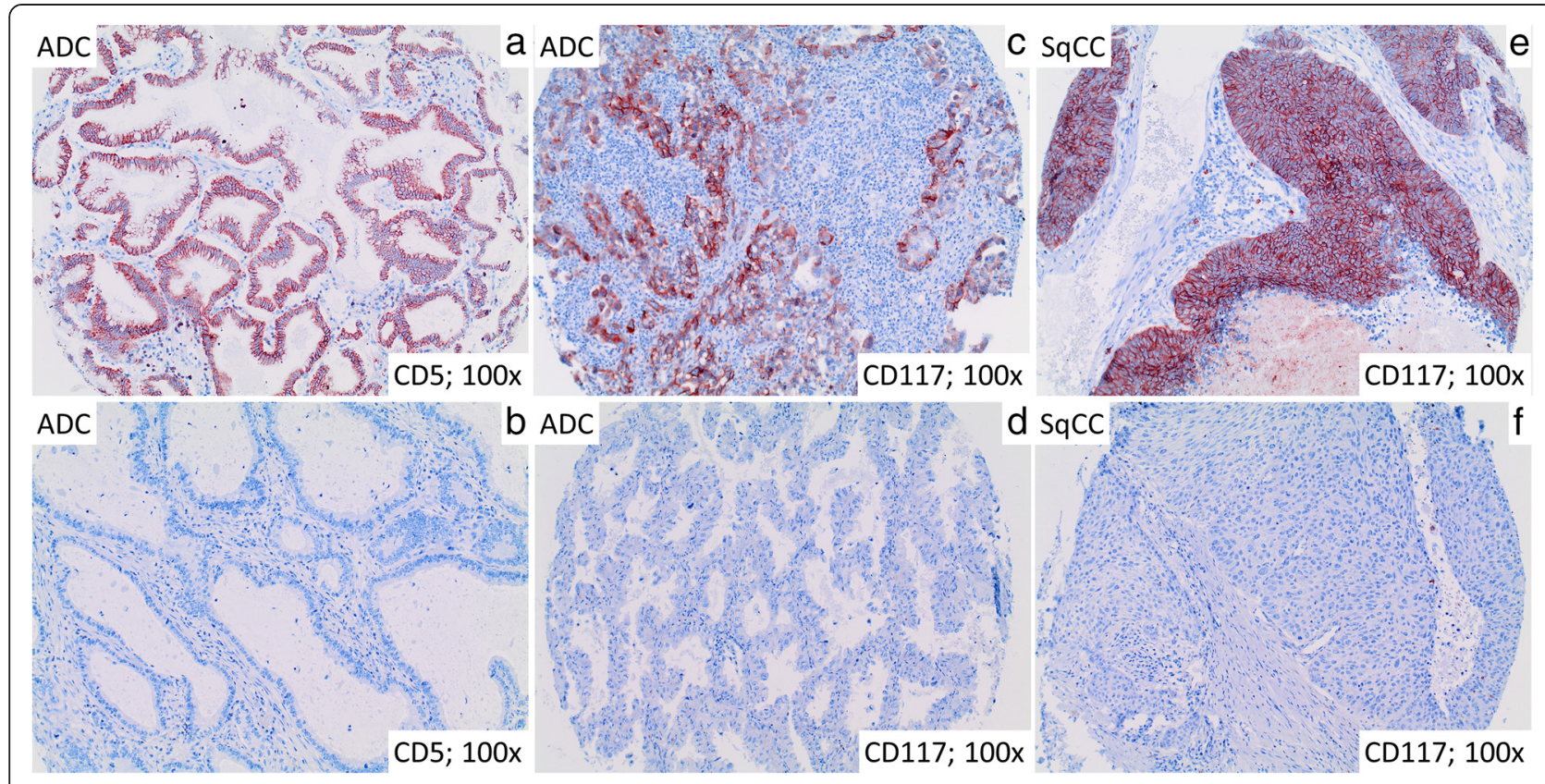

Fig. 1 Representative immunohistological stainings of CD117 and CD5 in ADC and SqCC. Representative images of ADC of the lung show CD5 (a) and CD117 (c) positive and negative cases (b, d). Moreover, a CD117 (e) positive and CD117 (f) negative SqCC is illustrated

primary lung and primary thymic squamous cell carcinomas with coexpression as a strong argument for a thymic primary.

Tumors of the mediastinum have a broad differential diagnosis ranging from benign disorders such as thyroid goiter or bronchogenic cysts to malignant diseases such as lymphoma, esophageal cancer or metastases from distant primaries [2]. Although routine thoracic imaging often initiate the subsequent evaluation of a mediastinal mass, it is rarely diagnostic and a biopsy is usually required to establish a definitive diagnosis. Albeit many entities have characteristic properties on H\&E staining, tumor subtyping remains challenging in some cases and additional IHC stainings are necessary for a final classification. Specifically in the setting of central thoracic neoplasms the differentiation of thymic carcinomas from NSCLC with mediastinal involvement can be difficult [12, 17]. Immunostaining against CD117, a

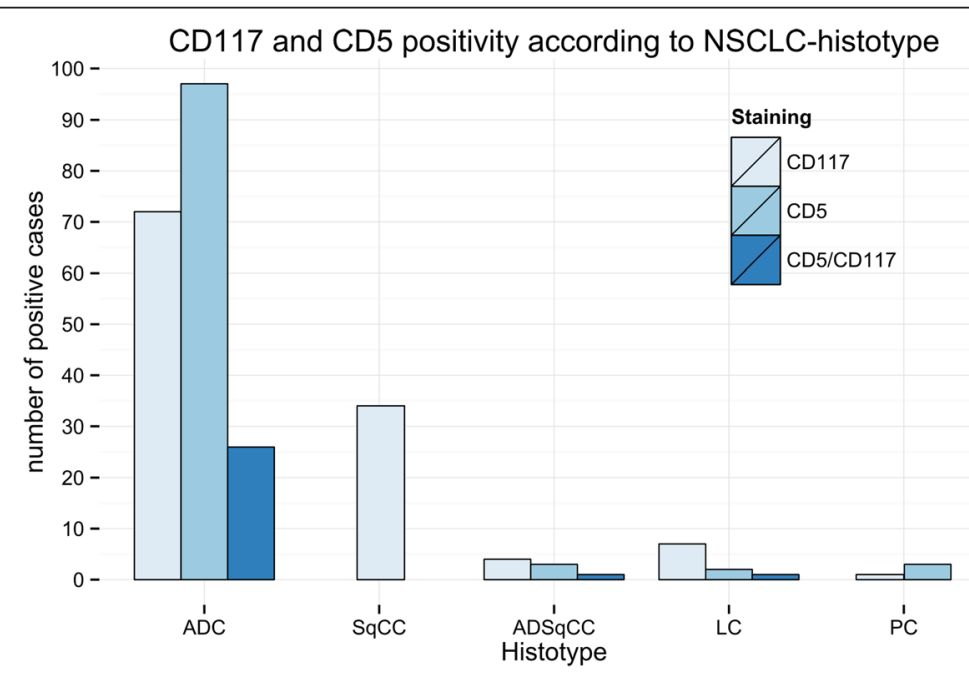

Fig. 2 CD117 and CD5 positivity according to NSCLC histotype. A barplot of the absolute number of CD117- (lightblue), CD5- (blue) and CD117/ CD5 (darkblue) positive NSCLC cases is shown according to the specific histotype. ADC: adenocarcinoma, SqCC: squamous cell carcinoma, ADSqCC: adeno-squamous carcinoma, LC: large cell carcinoma and PC: pleomorphic carcinoma 
transmembrane tyrosine kinase receptor, and against CD5, a member of the ancient scavenger receptor superfamily, is routinely performed to separate thymoma from thymic carcinoma. While CD117 is positive in approximately $85 \%$ of thymic carcinomas, CD5 is positive in approximately $70 \%$, and about $60 \%$ of cases show a CD117 and CD5 positive phenotype [8-10]. Thus, positivity of both markers support a diagnosis of thymic carcinoma. While several studies have investigated the expression of both markers in thymic carcinomas [8-12, 18-20], knowledge on the prevalence in large scale NSCLC cohorts is limited. Hence, we questioned if these markers might also be helpful in the differential diagnosis to NSCLC.

Albeit most studies revealed CD117 positivity in $20 \%$ $[9,18,21]$ of NSCLC cases, a wide range varying from $7 \%$ [22] to $64 \%$ [23] has been reported, mainly due to different cut-offs applied. Since positive or negative is the most important diagnostic aspect and cut-offs are usually not helpful in the interpretation of diagnostic IHC markers in small biopsies, we used a dichotomous scoring scheme. Moreover, CD117 has been suggested to be a prognostic marker for NSCLC. However, data is conflicting since some studies [22-24] demonstrated a prognostic effect of CD117 positivity in NSCLC but others could not confirm this finding [25]. In the present study we found CD117 in 145 out of 1457 (9.9\%) NSCLC which matches the reported range. Most CD117 positive tumors were ADC ( $66.9 \%$ of all CD117 positive cases), followed by SqCC (23.4 \%) which is well in line with the available literature [18].

CD5 has been investigated in NSCLC before and found to be present in $0-85 \%$ of cases [9, 10, 21]. CD5 positivity has been detected in a substantial subset of ADC in one study, but the results were limited by a rather small number of ADC investigated $(n=20)$ [21]. With regard to CD5 expression, a lack of representative large cohorts is also evident and the prevalence in NSCLC is unclear so far $[9,10,21]$. In this large scale study we found CD5 positivity in NSCLC in 133 out of 1427 NSCLC (9.2\%). Coexpression of CD117 and CD5 was detected in 28 out of 1465 cases (1.9\%) and was restricted to ADC, ADSqCC and LC. CD5 expression was not present in any of the $578 \mathrm{SqCC}$ cases. Thus, CD5 or CD117/CD5 coexpression in a upper mediastinal tumor is a strong argument for a thymic primary and argues against $\mathrm{SqCC}$ of the lung.

\section{Conclusions}

In summary, we demonstrate on 1465 NSCLC cases that a substantial subset exhibit CD117 and CD5 positivity and that pulmonary SqCC are consistently negative for CD5. Thus, the application of both immunomarkers is a valuable tool in the differential diagnosis of mediastinal tumors.

\section{Additional file}

Additional file 1: Table S1. Antibodies applied in this study.

(XLSX $10 \mathrm{~kb}$ )

\section{Competing interests}

The authors declare that they have no competing interests.

\section{Authors' contributions}

MK drafted the manuscript, participated in immunohistochemical analysis and data statistics. TM, TG and HD collected tissue material and revised the manuscript for important intellectual content. AH and LT participated in immunohistochemical analysis and data statistics. EH and AW designed the study and helped to draft the manuscript. All authors read and approved the manuscript.

\section{Acknowledgements}

Tissue samples were provided by the tissue bank of the National Center for Tumor Diseases (NCT, Heidelberg, Germany) in accordance with the regulations of the tissue bank and the approval of the ethics committee of Heidelberg University. MK was supported by the Postdoc Fellowship Programme of the Faculty of Medicine Heidelberg.

\section{Author details}

${ }^{1}$ Institute of Pathology, Heidelberg University, Im Neuenheimer Feld 224, Heidelberg, Germany. ${ }^{2}$ Translational Research Unit, Thoraxklinik at Heidelberg University, Heidelberg, Germany. ${ }^{3}$ Clinical and Experimental Pathology, Research Center Borstel, Borstel, Germany. 'Department of Thoracic Surgery, Thoraxklinik at Heidelberg University, Heidelberg, Germany. ${ }^{5}$ Translational Lung Research Center (TLRC), Member of the German Center for Lung Research, Heidelberg, Germany. ${ }^{6}$ Airway Research Center North (ARCN), Member of the German Center for Lung Research, Borstel, Germany.

Received: 19 September 2015 Accepted: 12 November 2015 Published online: 08 December 2015

\section{References}

1. Travis WD, Brambilla E, Burke AP, Marx A, Nicholson AG. WHO Classification of Tumours of the Lung, Pleura, Thymus and Heart. IARC; Lyon, France; 2015.

2. Yoneda KY, Louie S, Shelton DK. Mediastinal tumors. Curr Opin Pulm Med. 2001;7(4):226-33.

3. Huang J, Ahmad U, Antonicelli A, Catlin AC, Fang W, Gomez D, et al. Development of the international thymic malignancy interest group international database: an unprecedented resource for the study of a rare group of tumors. J Thorac Oncol. 2014;9(10):1573-8. doi:10.1097/JTO. 0000000000000269.

4. Hishida T, Nomura S, Yano M, Asamura H, Yamashita M, Ohde Y, et al. Long-term outcome and prognostic factors of surgically treated thymic carcinoma: results of 306 cases from a Japanese Nationwide Database Study. Eur J Cardiothorac Surg. 2015. doi:10.1093/ejcts/ezv239.

5. Syrios J, Diamantis N, Fergadis E, Katsaros L, Logothetis M, lakovidou I, et al. Advances in thymic carcinoma diagnosis and treatment: a review of literature. Med Oncol. 2014;31(7):44. doi:10.1007/s12032-014-0044-2.

6. Marx A, Rieker R, Toker A, Langer F, Strobel P. Thymic carcinoma: is it a separate entity? From molecular to clinical evidence. Thorac Surg Clin. 2011;21(1):25-31. doi:10.1016/j.thorsurg.2010.08.010. v-vi.

7. Marx A, Strobel P, Badve SS, Chalabreysse L, Chan JK, Chen G, et al. ITMIG consensus statement on the use of the WHO histological classification of thymoma and thymic carcinoma: refined definitions, histological criteria, and reporting. J Thorac Oncol. 2014;9(5):596-611. doi:10.1097/JTO. 0000000000000154.

8. Pan CC, Chen PC, Chiang H. KIT (CD117) is frequently overexpressed in thymic carcinomas but is absent in thymomas. J Pathol. 2004;202(3):375-81. doi:10.1002/path.1514. 
9. Nakagawa K, Matsuno Y, Kunitoh H, Maeshima A, Asamura H, Tsuchiya R. Immunohistochemical KIT (CD117) expression in thymic epithelial tumors. Chest. 2005;128(1):140-4. doi:10.1378/chest.128.1.140.

10. Asirvatham JR, Esposito MJ, Bhuiya TA. Role of PAX-8, CD5, and CD117 in distinguishing thymic carcinoma from poorly differentiated lung carcinoma. Appl Immunohistochem Mol Morphol. 2014;22(5):372-6. doi:10.1097/PAI. 0b013e318297cdb5.

11. Thomas de Montpreville V, Ghigna MR, Lacroix L, Besse B, Broet $P$, Dartevelle $P$, et al. Thymic carcinomas: clinicopathologic study of 37 cases from a single institution. Virchows Arch. 2013;462(3):307-13. doi:10.1007/ s00428-013-1371-y.

12. Pomplun S, Wotherspoon AC, Shah G, Goldstraw P, Ladas G, Nicholson AG. Immunohistochemical markers in the differentiation of thymic and pulmonary neoplasms. Histopathology. 2002;40(2):152-8.

13. Hishima T, Fukayama M, Fujisawa M, Hayashi Y, Arai K, Funata N, et al. CD5 expression in thymic carcinoma. Am J Pathol. 1994;145(2):268-75.

14. Tang ER, Schreiner AM, Pua BB. Advances in lung adenocarcinoma classification: a summary of the new international multidisciplinary classification system (IASLC/ATS/ERS). J Thorac Dis. 2014;6 Suppl 5:S489-501. doi:10.3978/j.issn.2072-1439.2014.09.12.

15. Terry J, Leung S, Laskin J, Leslie KO, Gown AM, lonescu DN. Optimal immunohistochemical markers for distinguishing lung adenocarcinomas from squamous cell carcinomas in small tumor samples. Am J Surg Pathol. 2010:34(12):1805-11. doi:10.1097/PAS.0b013e3181f7dae3.

16. Remmele W, Stegner HE. Recommendation for uniform definition of an immunoreactive score (IRS) for immunohistochemical estrogen receptor detection (ER-ICA) in breast cancer tissue. Pathologe. 1987;8(3):138-40.

17. Chung DA. Thymic carcinoma-analysis of nineteen clinicopathological studies. Thorac Cardiovasc Surg. 2000;48(2):114-9. doi:10.1055/s-2000-9868.

18. Butnor KJ, Burchette JL, Sporn TA, Hammar SP, Roggli VL. The spectrum of Kit (CD117) immunoreactivity in lung and pleural tumors: a study of 96 cases using a single-source antibody with a review of the literature. Arch Pathol Lab Med. 2004;128(5):538-43. doi:10.1043/1543-2165(2004)128<538: TSOKCl>2.0.CO;2.

19. Hayashi A, Fumon T, Miki Y, Sato H, Yoshino T, Takahashi K. The evaluation of immunohistochemical markers and thymic cortical microenvironmental cells in distinguishing thymic carcinoma from type b3 thymoma or lung squamous cell carcinoma. J Clin Exp Hematop. 2013;53(1):9-19.

20. Tsuchida M, Umezu H, Hashimoto T, Shinohara H, Koike T, Hosaka Y, et al. Absence of gene mutations in KIT-positive thymic epithelial tumors. Lung Cancer. 2008:62(3):321-5. doi:10.1016/j.lungcan.2008.03.035.

21. Saad RS, Landreneau RJ, Liu Y, Silverman JF. Utility of immunohistochemistry in separating thymic neoplasms from germ cell tumors and metastatic lung cancer involving the anterior mediastinum. Appl Immunohistochem Mol Morphol. 2003;11(2):107-12.

22. Yaren A, Oztop I, Kargi A, Ulukus C, Onen A, Sanli A, et al. Bax, bcl-2 and $c-k i t$ expression in non-small-cell lung cancer and their effects on prognosis. Int J Clin Pract. 2006:60(6):675-82. doi:10.1111/j.1368-5031.2006.00742.x

23. Micke $P$, Hengstler JG, Albrecht $H$, Faldum A, Bittinger F, Becker $K$, et al. c-kit expression in adenocarcinomas of the lung. Tumour Biol. 2004;25(5-6):235-42. doi:10.1159/000081386

24. Xiao H, Wang J, Liu Y, Li L. Relative influence of c-Kit expression and epidermal growth factor receptor gene amplification on survival in patients with non-small cell lung cancer. Oncol Lett. 2014;8(2):582-8. doi:10.3892/ol. 2014.2173.

25. Herpel E, Jensen K, Muley T, Warth A, Schnabel PA, Meister M, et al. The cancer stem cell antigens CD133, BCRP1/ABCG2 and CD117/C-KIT are not associated with prognosis in resected early-stage non-small cell lung cancer. Anticancer Res. 2011:31(12):4491-500.

\section{Submit your next manuscript to BioMed Central and take full advantage of:}

- Convenient online submission

- Thorough peer review

- No space constraints or color figure charges

- Immediate publication on acceptance

- Inclusion in PubMed, CAS, Scopus and Google Scholar

- Research which is freely available for redistribution

Submit your manuscript at www.biomedcentral.com/submit 